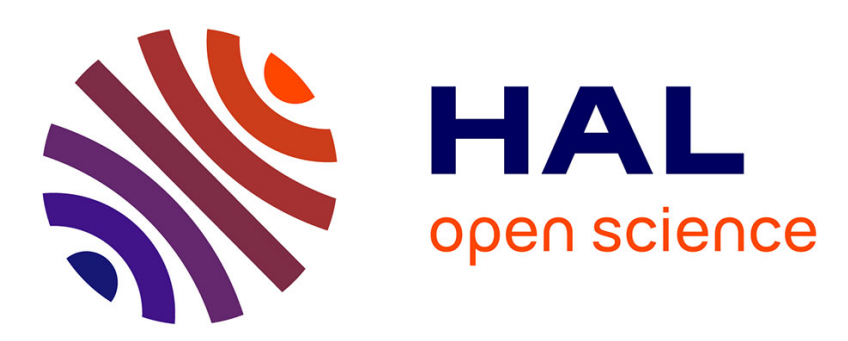

\title{
Utilisation d'un système d'information géographique pour l'élaboration d'un schéma de desserte exemple d'application sur le massif de melles (haute-garonne) \\ Véronique Chéret, M. Gay
}

\section{- To cite this version:}

Véronique Chéret, M. Gay. Utilisation d'un système d'information géographique pour l'élaboration d'un schéma de desserte exemple d'application sur le massif de melles (haute-garonne). Revue forestière française, 1995, 67 (6), pp.647-660. 10.4267/2042/26688 . hal-03444279

\author{
HAL Id: hal-03444279 \\ https://hal.science/hal-03444279
}

Submitted on 23 Nov 2021

HAL is a multi-disciplinary open access archive for the deposit and dissemination of scientific research documents, whether they are published or not. The documents may come from teaching and research institutions in France or abroad, or from public or private research centers.
L'archive ouverte pluridisciplinaire HAL, est destinée au dépôt et à la diffusion de documents scientifiques de niveau recherche, publiés ou non, émanant des établissements d'enseignement et de recherche français ou étrangers, des laboratoires publics ou privés. 


\title{
technique et forêt
}

\section{UTILISATION D'UN SYSTÈME D'INFORMATION GÉOGRAPHIQUE POUR L'ÉLABORATION D'UN SCHÉMA DE DESSERTE Exemple d'application sur le massif de Melles (Haute-Garonne)}

\author{
Véronique CHÉRET - M. GAY
}

Dans l'article de P. de Meerleer paru dans le $n^{\circ}$ 5/1995 de la Revue forestière française sous le titre "Mobilisation des bois dans un massif de montagne", l'Office national des Forêts présente la mise au point d'une mèthode d'établissement d'un schéma de desserte. L'étude a portė sur un massif boisè de montagne (Melles) où les contraintes environnementales (faunistiques et floristiques) et techniques d'exploitation sont fortes.

La méthode intègre tout un ensemble d'informations à caractère géographique et repose sur une estimation quantitative :

- d'une part, des contraintes du milieu naturel,

- d'autre part, des données économiques liées à l'exploitation des bois par la création d'un réseau de desserte (routes, pistes ou câbles) adapté aux caractéristiques du massif.

Grâce à ce procédé mis au point, le gestionnaire forestier peut être renseigné, pour un massif donné, sur l'intérêt économique de développer une voirie forestière. Il peut surtout faire son choix sur le type de desserte à créer pour optimiser le rendement, tout en respectant les caractéristiques environnementales du site.

Une application directe de la méthode à n'importe quel massif forestier, dans un but à la fois d'aménagement et de protection des biotopes, est envisageable. Quand on connaît les coûts qu'occasionne la construction d'une voirie forestière et le montant des subventions accordées pour celle-ci, on comprend aisément que la méthode ainsi constituée est à même de devenir un outil permettant une meilleure prise de décision, tant par les gestionnaires forestiers que par les décideurs publics. 
C'est en considérant le caractère géographique de l'information utilisée et le principe de manipulation de données cartographiques que le modèle est apparu généralisable à un Système d'Information géographique (SIG). Le SIG est un outil qui permet de traiter de l'information spatialisée pour le stockage de données, avec surtout la possibilité de recoupement de données d'origines diverses et la création d'informations nouvelles. L'intérêt de son utilisation dans la gestion forestière, à différentes échelles : région, massif, forêt, parcelle..., a déjà été montré.

Dans le cadre d'une action coordonnée par le Conseil régional de Midi-Pyrénées pour la mise en place d'une Stratégie forestière, il nous a donc été demandé de prolonger la mise au point de la méthode ONF par son intégration sous la forme d'un SIG "d'aide à la constitution d'un schéma de desserte".

Le développement de cette méthode s'est fait en concertation avec la Direction régionale de l'ONF pour la région Midi-Pyrénées.

FONCTIONNEMENT OU SIG

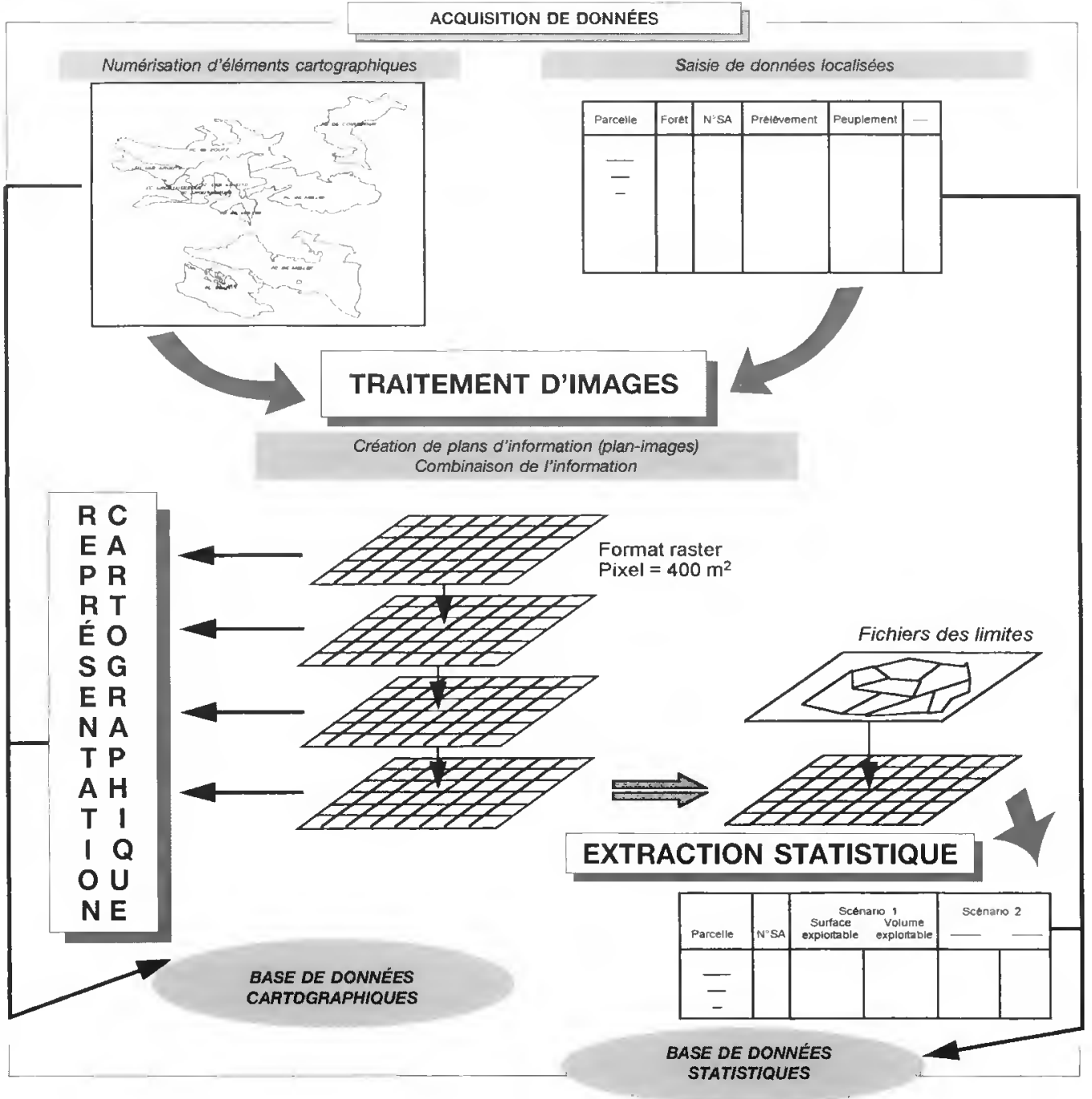


Le but fixé à ce travail se situe à plusieurs niveaux :

- vérifier que les deux démarches, l'une (de l'ONF) utilisant des procédés cartographiques manuels et celle que nous avons expérimentée à l'aide d'outils informatiques, aboutissaient à des résultats similaires;

- mesurer les possibilités offertes, par les moyens informatiques mis en jeu, de modifier à volonté les paramètres pour une meilleure adaptation du modèle;

- montrer que le SIG permet d'obtenir des informations complémentaires susceptibles d'intéresser les gestionnaires forestiers ;

- rendre plus aisée la généralisation du modèle pour une application à d'autres massifs.

\section{MISE EN PLACE DE DONNÉES DANS LE SIG. MODE DE TRAITEMENT DE L'INFORMATION}

\section{Contexte technique}

Le SIG a été développé autour de quatre points : l'acquisition de données spatialisées, le traitement de l'information sous système de traitement d'images, l'extraction de données statistiques, et enfin la représentation cartographique des données de base et de synthèse.

Des précisions sur la méthode et les outils utilisés sont présentées en annexe (p. 659).

Le schéma général d'exploitation du SIG est donné par la figure 1 (p. 648).

\section{Données intégrées dans le SIG}

\section{- Cartes thématiques}

Toutes les informations cartographiques utilisées par l'ONF ont été numérisées à partir de cartes analogiques couvrant le massif de Melles. Elles ont servi à constituer, en mode raster, les planimages de base nécessaires à l'analyse spatiale. L'unité élémentaire de ces plans d'information (pixel) a pour résolution $400 \mathrm{~m}^{2}$.

\begin{tabular}{|c|c|c|c|}
\hline Type de données & Contenu des cartes & Source & Échelle \\
\hline Données foncières & $\begin{array}{l}\text { Limites de forêts (propriétè) } \\
\text { Parcellaire forestier }\end{array}$ & $\begin{array}{l}\text { ONF } \\
\text { ONF }\end{array}$ & $\begin{array}{l}1 / 10000 \\
1 / 10000\end{array}$ \\
\hline Données environnementales .... & $\begin{array}{l}\text { Peuplements forestiers } \\
\text { Topographie : numérisation des cour- } \\
\text { bes de niveau équidistantes de } \\
50 \text { mètres pour la constitution d'un } \\
\text { modèle numérique de terrain et no- } \\
\text { tamment du plan d'information des } \\
\text { pentes. } \\
\text { Géologie } \\
\text { Faune (zonage Ours, Grand Tétras } \\
\text { et lsard) }\end{array}$ & $\begin{array}{c}\text { IFN - ONF } \\
\text { IGN }\end{array}$ & $\begin{array}{l}1 / 80000 \\
1 / 10000\end{array}$ \\
\hline Données forestières. & $\begin{array}{l}\text { Prévision des récoltes pour les } 20 \text { ans } \\
\text { à venir (prélèvement) } \\
\text { Réseau de desserte existant (routes } \\
\text { et pistes forestières) } \\
\text { Secteurs d'analyse (au sens ONF) } \\
\text { Surfaces exploitables } \\
\text { (pour les } 3 \text { scénarios) }\end{array}$ & $\begin{array}{l}\text { ONF } \\
\text { ONF } \\
\text { ONF } \\
\text { ONF }\end{array}$ & $\begin{array}{l}1 / 10000 \\
1 / 10000 \\
1 / 10000 \\
1 / 10000\end{array}$ \\
\hline
\end{tabular}




\section{- Données localisées}

Elles sont principalement relatives aux données forestières (prélèvements en $\mathrm{m}^{3}$ de bois à l'hectare, composition en essences des peuplements...) et associées aux contours des parcelles, limites de peuplements, secteurs d'analyse.

\section{EXPLOITATION DU SIG}

Traitement des images.

Analyse des contraintes du massif forestier de Melles

Pour suivre le protocole établi par l'ONF, la première phase correspond à une quantification des contraintes que l'on pourrait qualifier de "contraintes élémentaires". Elles sont au nombre de trois :

- contrainte environnementale ou contrainte faune, liée surtout aux biotopes (Ours et Grand Tétras) à protéger ;

- contrainte technique ou contrainte relief, la création d'une voirie étant techniquement dépendante de la nature du substrat et de la topographie;

- contrainte économique ou contrainte prélèvement, l'intérêt de mobiliser du bois étant avant tout lié au volume de bois récoltable.

Pour cette première étape, les quatre plan-images de base utilisés ont été élaborés à partir des informations : faune, géologie, pente et prélèvement, préalablement mises en place dans le SIG.

Les plan-images de contraintes élémentaires ont été obtenus (figure 2 - phase 1) par attribution d'une valeur de contrainte à chaque valeur thématique des plan-images de base. La correspondance est la suivante :

\begin{tabular}{|c|c|c|c|c|c|}
\hline Faune & $\begin{array}{l}\text { Poids de } \\
\text { contrainte }\end{array}$ & Relief & $\begin{array}{l}\text { Poids de } \\
\text { contrainte }\end{array}$ & $\begin{array}{l}\text { Prélèvement } \\
\text { en } \mathrm{m}^{3} / \mathrm{ha}\end{array}$ & $\begin{array}{l}\text { Poids de } \\
\text { contrainte }\end{array}$ \\
\hline 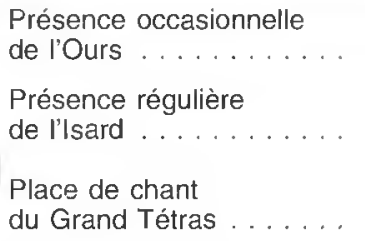 & 10 & $\begin{array}{l}\text { pente }<45 \% \\
\text { terrains stables }\end{array}$ & 5 & $\mathrm{P}>70$ & 0 \\
\hline $\begin{array}{l}\text { Présence régulière } \\
\text { de l'Ours } \ldots \ldots \ldots \ldots\end{array}$ & 20 & $\begin{array}{l}\text { pente } 45-75 \% \text { el } \\
\text { terrains peu stables }\end{array}$ & 15 & $40<\mathrm{P}<70$ & 20 \\
\hline $\begin{array}{l}\text { Site vital et centre d'activité } \\
\text { de l'Ours } \ldots \ldots \ldots \ldots\end{array}$ & 30 & $\begin{array}{l}\text { pente }>75 \% \\
\text { ravins très encaissés } \\
\text { terrains instables }\end{array}$ & 30 & $P<40$ & 30 \\
\hline
\end{tabular}

Comme l'indique la figure 2 (phase 1), la contrainte relief résulte de la combinaison de la pente et de la géologie avec attribution "conditionnelle" d'un poids de contrainte ; exemple : la valeur 30 (forte contrainte) est affectée aux pixels ayant pour valeur thématique "pente de plus de $75 \%$ et/ou ravin très encaissé" dans le plan pente, et/ou "terrain instable" dans le plan géologie.

La deuxième phase consiste à cartographier le poids de contrainte totale (figure 2 - phase 2). II est directement obtenu par la combinaison des trois plan-images des contraintes élémentaires et par sommation des poids de contrainte selon le modèle ONF. 


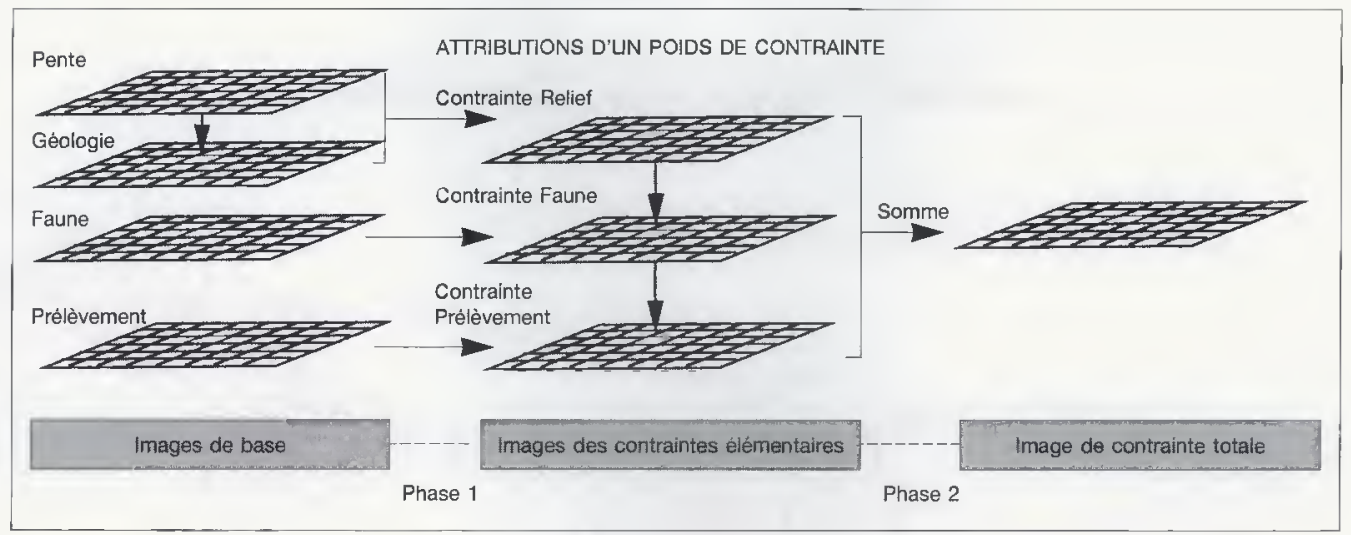

Contrairement à la méthode ONF, la quantification, ainsi que la cartographie, des poids de contrainte sur l'ensemble du massif se font sans découpage en unités et secteurs d'analyse, l'entité renseignée étant le pixel $\left(400 \mathrm{~m}^{2}\right)$. La carte obtenue (figure 3, p. 652) fait particulièrement bien ressortir les variations du poids de contrainte totale intramassif et même intraparcellaire, la combinaison de l'information se faisant de pixel à pixel et donc indépendamment des limites d'unités de gestion.

On comprend aisément que le procédé d'utilisation de plans numériques laisse à tout moment la possibilité de faire varier le poids des contraintes élémentaires, et même de rajouter ou d'éliminer une de ces contraintes. Cela peut en effet être le cas si l'on veut, par exemple, augmenter le poids de la contrainte Ours (en rapport avec le projet de réintroduction d'individus dans le massif) ou au contraire si l'on décide de ne plus la prendre en considération dans le projet d'aménagement du massif.

Une autre possibilité offerte par le système est le croisement de chaque plan-image de contrainte élémentaire avec celui du poids de contrainte totale. On obtient ainsi une cartographie des contraintes dites "relatives" où les valeurs sont exprimées en $\%$ de poids total. On peut par exemple faire ressortir les secteurs où la contrainte Ours représente plus de $60 \%$ du poids total de contrainte ou, au contraire, les secteurs où la contrainte relief prédomine largement.

\section{Extractions statistiques}

Le massif de Melles a été découpé en 14 secteurs d'analyse ou "unités de gestion élémentaires" à l'intérieur desquelles la desserte peut être envisagée de façon autonome.

L'utilisation de "l'arbre des choix de desserte", tel qu'il a été défini par l'ONF, suppose que l'on dispose du poids des contraintes (élémentaires et totale) de chaque secteur d'analyse, ainsi que le niveau moyen des contraintes de l'ensemble du massif pour l'établissement de seuils de comparaison. Ceci est rendu directement possible, et automatisable, par croisement du fichier vecteur des limites de secteur d'analyse (préalablement numérisées) avec les différents plan-images de contraintes (figure $4, p .653$ ). Les résultats obtenus sont directement pondérés par la surface (connaissant la résolution du pixel). Ils sont intégrés et réorganisés dans la base de données. En complément d'information, on peut procéder de la même manière en utilisant les limites du parcellaire forestier ou des forêts.

De la même manière, il était nécessaire d'extraire le volume de bois potentiellement exploitable selon trois scénarios de desserte possibles. 
Figure 3

POIDS DE CONTRAINTE TOTALE

(CONTRAINTE FAUNE + CONTRAINTE RELIEF + CONTRAINTE PRÉLÈVEMENT)

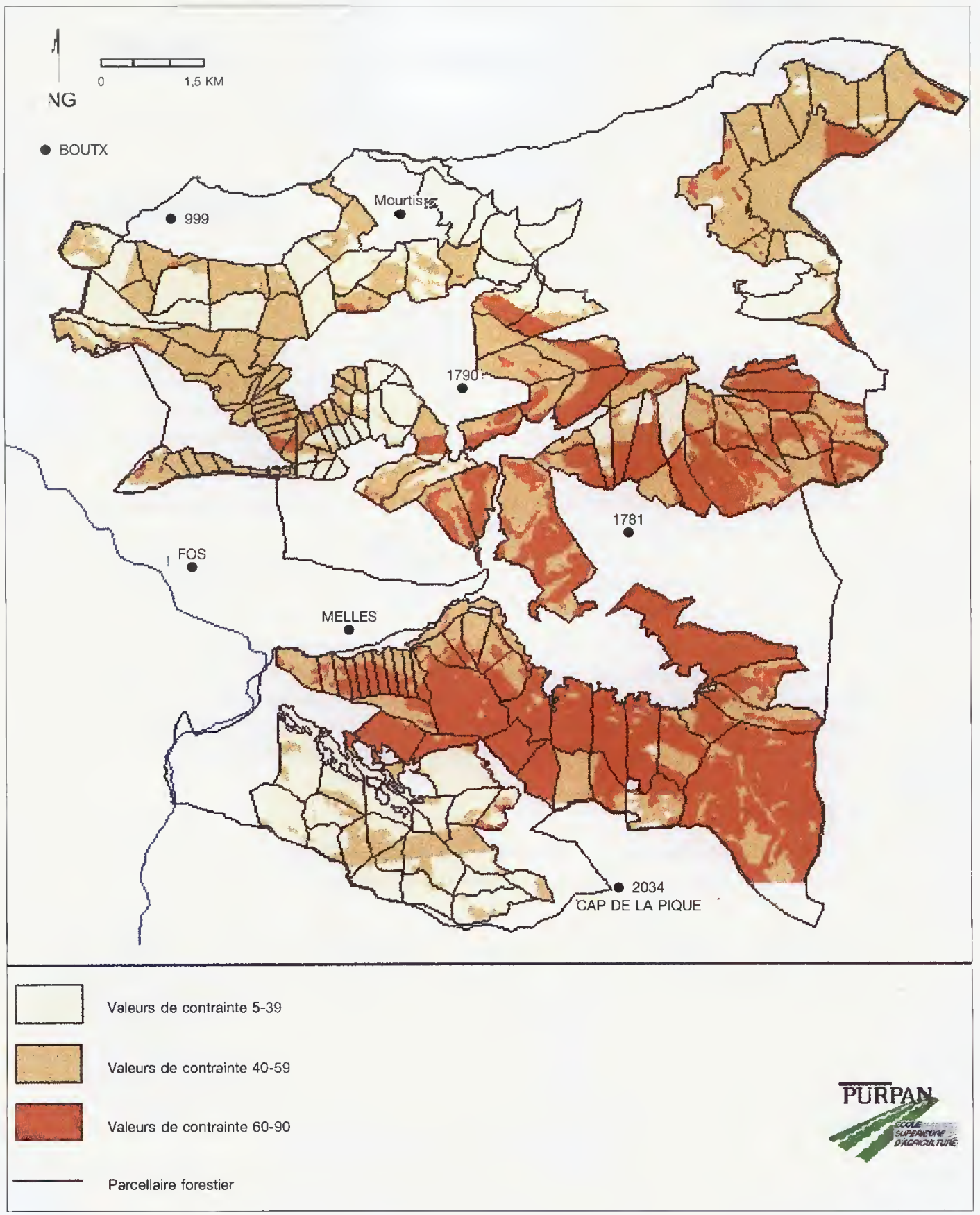


En effet, l'ONF a retenu trois modes de desserte réalisables sur le massif de Melles : le débardage par routes et pistes (scénario 1), par routes et câbles courts (scénario 2), par routes et câbles longs (scénario 3).

Pour chacun des trois scénarios, et pour les secteurs où leur mise en œuvre était possible indépendamment de l'analyse des contraintes précédentes, l'ONF a établi le tracé de la desserte à créer, et a délimité les surfaces forestières potentiellement exploitables.

À partir de cette information cartographique, nous avons procédé à l'estimation des volumes de bois mobilisables. Le calcul a été fait directement par croisement du plan des prélèvements avec celui des surfaces exploitables par scénario (figure 4). On intègre ainsi dans la base de données un volume $\left(\mathrm{m}^{3}\right)$ de bois récoltable dans chaque secteur d'analyse et pour chaque scénario de desserte.

La récolte potentielle est la seule donnée économique ayant été intégrée dans la base de données.

Figure 4

\section{EXTRACTIONS STATISTIQUES}

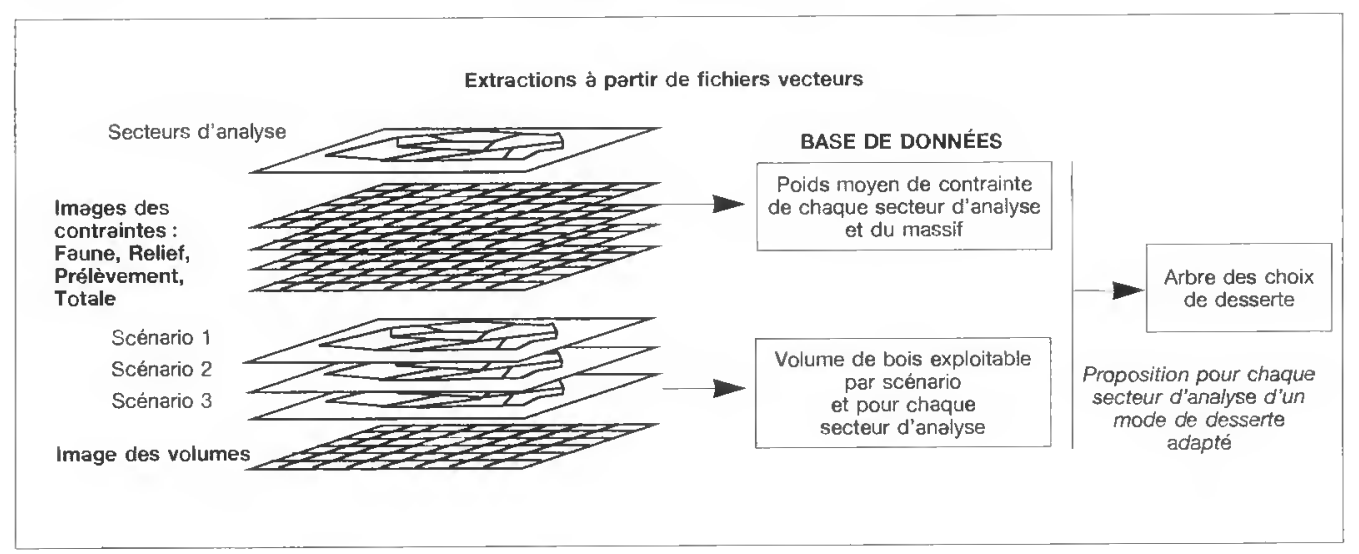

\section{CHOIX D'UN MODE DE DESSERTE}

\section{Utilisation de "l'arbre des choix de desserte"}

L'étape finale de l'étude est de proposer, pour chaque secteur d'analyse, le mode de desserte le mieux adapté possible compte tenu des contraintes analysées et des volumes potentiellement exploitables.

Les deux types d'information obtenue, contrainte et volume exploitable, permettent ainsi d'utiliser l'itinéraire arborescent élaboré par l'ONF (figure 5, p. 654). À chaque palier de cet arbre, une valeur "seuil" de contrainte (niveau moyen du massif) induit une distribution des secteurs d'analyse en fonction de leur propre poids de contrainte. Le dernier niveau de distribution correspond à une comparaison des volumes exploitables puis à la proposition d'un type de desserte approprié à chaque secteur d'analyse. On retiendra en final le scénario de desserte (Sc1, Sc2, Sc3) pour lequel le volume exploitable est le plus important.

Contrairement au modèle ONF, les données financières (soldes) n'ont pas été intégrées dans l'analyse. Toutefois, les résultats obtenus sont très proches :

- Lorsque le poids de contrainte totale est supérieur à 60 (seuil maximum fixé par l'ONF), aucune desserte n'est proposée. 
Figure 5 
Les deux procédés sont donc fondés sur la saisie et l'exploitation des mêmes données de base, toutes de nature gėographique. La similitude des résultats montre que l'introduction d'une approche de type SIG ne modifie pas fondamentalement la manière de collecter l'information, et de la traiter.

Dans la méthodologie ONF, toutes les informations utilisées doivent être contenues dans les mêmes unités de surface, sur des supports cartographiques de même échelle et sous la forme de valeurs moyennes (définition préalable d'unités et de secteurs d'analyse). II existe donc une dépendance entre les plans d'information qui d'une part, limite le recours à des informations non disponibles à l'échelle de travail prédéfinie, et d'autre part, réduit les possibilités de calcul aux seules données qui sont communes à toutes les entités.

L'utilisation de la technique SIG élimine complètement ces restrictions :

- Les données à saisir peuvent être issues de cartes d'échelle différente, I'homogénéité dans le fonctionnement du SIG étant assurée par la référence cartographique commune adoptée pour toutes les données intégrées.

- Les informations ne doivent pas obligatoirement être contenues dans les mêmes limites.

- Les plans, composés de pixels ayant chacun une valeur propre, sont indépendants les uns des autres et superposables (cf. figure 1, p. 648).

Les plans de synthèse, résultant de différents croisements, ont une précision qui dépend de celle de la plus mauvaise donnée initiale. Le fait de ne disposer d'information sur la géologie que par le biais de la carte au $1 / 80000^{\mathrm{e}}$ limite la qualité des plans dans lesquels cette donnée sera introduite. En dehors de cette restriction, cet outil s'adapte très bien à une telle analyse du territoire.

\section{Apport du SIG}

\section{- Adaptation et généralisation du modèle}

Cette étude de faisabilité montre la possibilité de généraliser le modèle pour l'appliquer à d'autres massifs forestiers, en en faisant un véritable outil d'analyse et de choix de desserte. Par rapport à la méthode manuelle, l'intérêt fondamental du SIG est la facilité qu'il offre à intégrer à tout moment de nouvelles données spatialisées ou de modifier les cotations de contrainte pour refaire automatiquement l'analyse.

\section{- Le choix des contraintes et leur cotation peuvent être modifiés}

Dans l'étude de Melles, la contrainte environnement joue un rôle important pour l'établissement du schéma de desserte. Elle reste essentiellement liée au zonage Ours. On peut améliorer l'estimation de la sensibilité écologique par la prise en compte de donnèes environnementales supplémentaires (données des ZNIEFF par exemple).

On peut éventuellement être amené à intégrer d'autres types d'information tels que des aménagements ou projets touristiques.

Un travail méthodologique peut être engagé pour mieux aborder les calculs concernant la notion même de contrainte. Deux pistes se dégagent : la contrainte totale résulte ici d'un processus de sommation simple. II est tout à fait envisageable d'introduire des notions de condition qui, pour une situation donnée, imposeraient une contrainte totale donnée, indépendamment des valeurs des autres plans d'information. Ces techniques sont bien maîtrisées par les outils SIG.

La seconde piste demande un développement plus complexe: il s'agit d'aborder la définition des valeurs seuils de contrainte, non pas comme moyenne de leurs valeurs sur l'ensemble du massif, mais comme résultant d'un processus itératif. Dans ce processus, on chercherait à définir la valeur seuil d'une contrainte en fonction d'un coût économique ou d'une conséquence économique. Par exemple, la modification de 10 points de la valeur seuil pour la contrainte environnement a une conséquence économique qui peut être calculée. 
Figure 6

SCÉNARIOS D'EXPLOITATION RETENUS

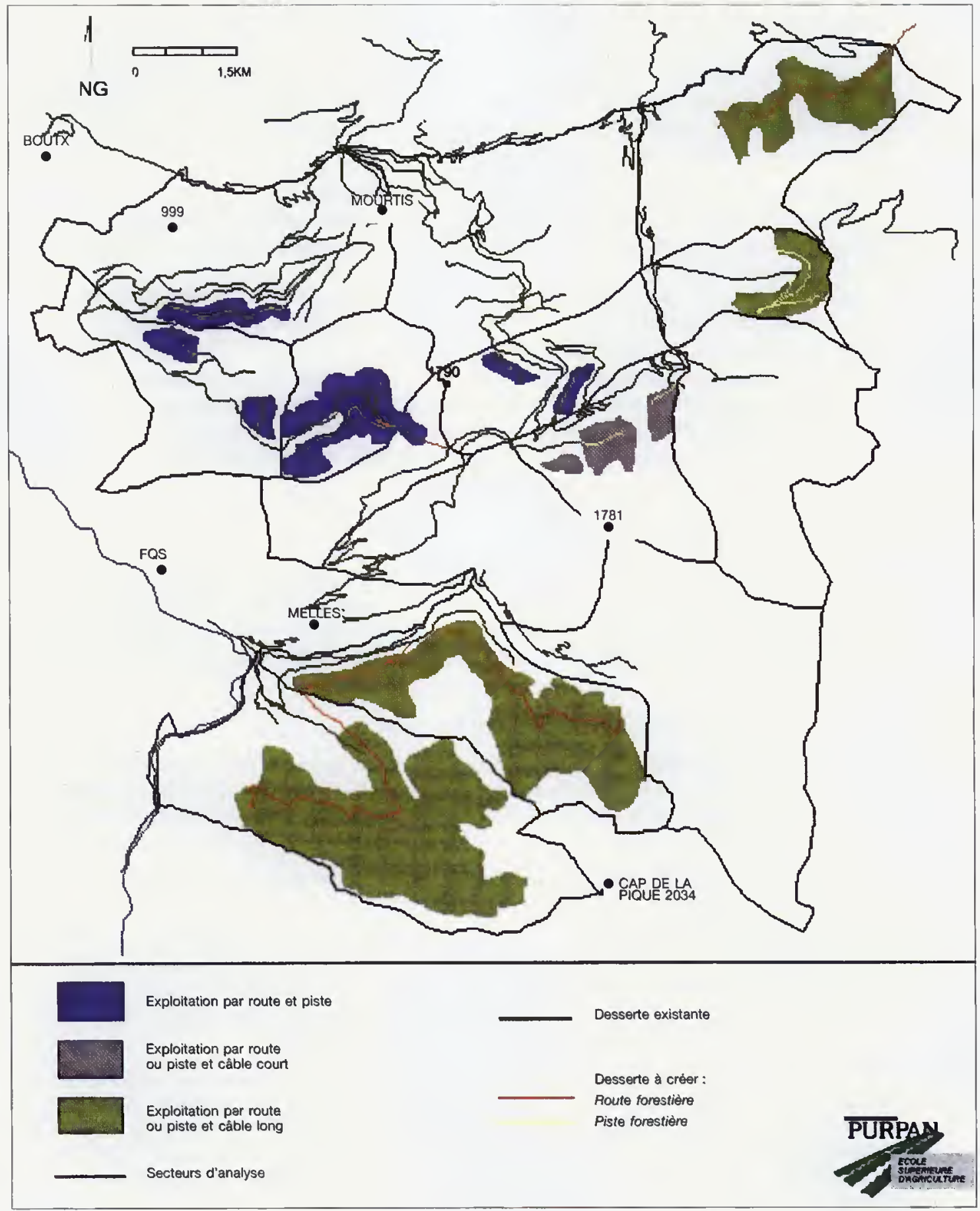


- Une réactualisation des données de base serait facilement réalisable

A la différence de l'approche manuelle, la technique du SIG permet de mettre en place une analyse dynamique d'un massif par la possibilité de réactualiser les données de base et de refaire automatiquement l'analyse. Dans l'état actuel de notre réflexion, une telle utilisation pourrait être envisagée pour des actions comme :

- la nécessité de modifier la surface exploitable à la suite d'un coup de chablis ou d'un problème phytosanitaire. De telles modifications des contraintes d'exploitation nécessitent une réactualisation rapide des données tant au niveau de la carte que de la base de données;

- une modification des conditions d'environnement (changement de la réglementation pour la protection d'une espèce par exemple) peut imposer une nouvelle analyse des conditions d'exploitation du massif.

\section{- Le SIG permet la restitution cartographique de toutes les données}

Une caractéristique importante de l'utilisation de la technique SIG est de rendre l'accès au document cartographique particulièrement aisé. Chaque donnée introduite dans le système fournit une information visualisable sur tout l'espace. Le résultat est particulièrement intéressant lorsque l'on arrive à proposer un document de synthèse, qui n'existait pas jusqu'alors ou non réalisable manuellement, par exemple la cartographie de la contrainte totale à la résolution de $400 \mathrm{~m}^{2}$, ou des contraintes relatives.

\section{- Un outil d'inventaire et de gestion}

Par le croisement de plans d'information avec les limites des unités de gestion, une base de données est alimentée en informations non graphiques relatives au contenu des parcelles.

Cette seconde caractéristique doit être rapprochée de l'intérêt des SIG en tant qu'outil de gestion du territoire. Le fait de disposer d'unités de gestion (parcelles forestières, limites de forêt ou de massif), associées à des procédés d'extraction, permet d'exploiter statistiquement les données disponibles dans les différents plans d'information. Ce procédé n'est dépendant ni de la taille des pixels, ni des limites des entités de chacun des plans étudiés.

De cette manière, la composition en essences, les volumes et les surfaces exploitables selon les différents scénarios de desserte ont été évalués de manière précise pour chaque parcelle. À l'inverse, une information parcellaire, calculée à partir de traitements effectués dans la seule base de données, peut parfaitement être restituée sous forme cartographique.

Les possibilités d'exploitation, centrées sur la base de données, sont multiples et peuvent être utilisées pour renseigner le propriétaire de la parcelle, de la forêt ou le gestionnaire du massif.

Enfin, l'introduction progressive des informations résultant de l'exploitation du massif (localisation et date des coupes, volume prélevé, estimation du volume résiduel et état du boisement, etc.) permettra de faire vivre cette base de données en lui conférant une valeur d'outil de gestion, grâce à l'actualisation permanente des données qu'elle contient.

\section{- Tracé automatique de la desserte}

Le logiciel de SIG que nous avons utilisé ne disposait pas des possibilités de calcul de tracé automatique de routes et pistes en fonction de contraintes de direction, de pente ou de nature géologique des sols traversés. Il nous semble envisageable de concevoir des outils pour améliorer les informations sur certains points déterminant le coût de construction des routes, comme la distance à parcourir, mais aussi des notions de pente en travers, talus, proportion de rochers, qui permettent de définir les cubages de déblais à déplacer. 
II n'est pas envisageable de basculer pour autant dans le "tout automatique". La montagne est un milieu complexe dans lequel le choix d'un tracé repose sur un grand nombre d'éléments, à caractère souvent qualitatif. Comme un SIG ne pourra jamais prendre en compte la totalitè de l'expertise des hommes sur le terrain, il ne peut fournir que des informations incomplètes sur la localisation de ces tracés. Par contre, sa capacité d'analyse systématique de toutes les solutions possibles peut amener à proposer des tracés qui n'avaient simplement pas été envisagés.

\section{CONCLUSIONS}

Ce travail a mis en évidence que l'investissement engagé dans la création d'une base de données à caractère géographique, tel que cela a été fait sur le massif de Melles, peut être valorisé :

- par l'aide qu'il apporte dans la définition de la stratégie de desserte;

- en tant que mise au point d'un modèle généralisable à d'autres massifs forestiers, en laissant la possibilité d'adapter finement le procédé par la prise en compte des contraintes spécifiques de chaque milieu ètudié ;

- par la démonstration des possibilités d'entretenir la base de données (cartographiques et statistiques) pour suivre la vie et l'évolution d'une forêt, inventorier et gérer les ressources forestières.

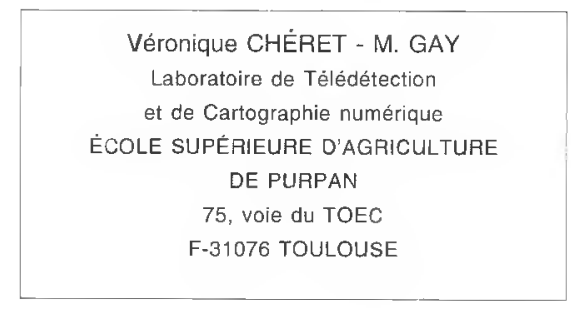




\section{ANNEXE \\ Mise en place et fonctionnement du SIG}

\section{- Acquisition des données}

Numérisation de cartes : la première étape a consisté à intégrer dans la base de données toute l'information fournie sous forme de cartes thématiques.

Les éléments cartographiques ont été numérisés à l'aide d'un logiciel de numérisation (SICIS) implanté sur station VAX VMS, et référencés dans un même système de projection cartographique (Lambert III). Les fichiers ainsi obtenus sont au format "vecteur".

Saisies de données localisées : des informations forestières localisées, au niveau des parcelles ou de limites de peuplements, ont été intégrées dans la base de données. La saisie et l'organisation se sont faites sous logiciel de gestion de base de données relationnelles (DBASE IV) sur PC.

\section{- Traitement d'images}

Compte tenu de la nature de l'information à manipuler et du type de croisement à effectuer (combinaison, fusion, superposition), nous avons choisi de travailler en mode "raster".

Pour cela, à l'aide des fichiers issus de la numérisation des cartes et des données descriptives associées aux entités géographiques de gestion (parcelles, peuplements...), nous avons constitué tout un ensemble de planimages, sous logiciel de traitement d'images (ANAIS) implanté sur station VAX VMS.

Par un jeu de points d'appui, les plan-images sont rendus tous parfaitement superposables. La résolution des points élémentaires (pixels) est de $400 \mathrm{~m}^{2}$.

\section{- Extraction de données statistiques}

Un logiciel taisant l'interface Traitement d'images - Logiciel de gestion de base de données relationnelles (DBASE IV) nous a permis d'extraire, à partir des fichiers des contours (vecteurs), le contenu en information des plan-images. Généralement, l'information à extraire se rapportait à des valeurs de surfaces et volumes exploitables, ou au poids de contrainte des secteurs d'analyse.

\section{- Représentation cartographique}

Toutes les informations référencées qui ont été mises en place sous le traitement d'images peuvent être restituées sous forme cartographique, qu'il s'agisse des plans d'information de base utilisés pour l'analyse (pente, géologie, faune, type de peuplement...) ou des résultats issus des divers croisements réalisés (contraintes élémentaires, contrainte totale du massif...).

Pour simplifier les modalités de sorties cartographiques et pour les données qui s'y prêtaient, certains planimages (en mode raster) ont été polygonisés pour constituer des "couvertures" (en mode vecteur) sous logiciel ARC/NFO sur PC. 


\section{BIBLIOGRAPHIE}

ARCHAMBAULT (L.), BEDARD (Y.). - Geomatique et SIRS en foresterie. - Formation continue, cours $\mathrm{n}^{\circ} 17$. - Ordre des Ingénieurs forestiers du Québec (Canada), 1990. - $10 \mathrm{p}$.

CHÉRET (V.), GAY (M.). - Stratégie forestière et information géographique. Mise en place d'un outil d'aide à la décision pour l'établissement d'une stratégie forestière régionale. - Rapport de contrat $n^{\circ} 961 / 657 / 73$. - $18 \mathrm{p}$. et annexes.

DE MEERLEER (P.), MAGRUM (M). - Ėtude expérimentale de mobilisation des bois dans un massif boisė de montagne. Massif de Melles (Haute-Garonne), — Office national des Forêts, 1992. - 34 p. (Rapport interne).

JOLLY (A.), GUYON (D.). - Possibilités de suivi par un système d'information gèographique de la gestion des forêts traitèes en futaie régulière. - Revue forestière française, vol. XLV, n spécial "Informatique et Foresterie", 1993, pp. 108121.

SERRA (E.), THUM (P.), VENTURA (S.), NIEMANN (B.). - Evaluating the relationships between agricultural forest management and environnemental quality using a multipurpose LIS. - American congress on surveying and mapping ALSM, annual Convention 1990, vol. 3, GIS/LIS, USA, pp. 261-269. 